Agronomie

\title{
Diagnostic de la nutrition minérale de prairies permanentes au printemps. I. Établissement de références
}

\author{
M Duru \\ INRA, centre de recherches de Toulouse, station d'agronomie, BP27 - 31326 Castanet-Tolosan, France
}

(Reçu le 10 octobre 1990; accepté le 16 janvier 1992)

avec la collaboration de $\mathrm{L}$ Sos et $\mathrm{R}$ Viard

\begin{abstract}
Résumé - Les cinétiques d'accumulation de l'azote, du phosphore et du potassium en fonction de la production de biomasse ont été comparées pour la pousse de printemps de 4 prairies permanentes. Ces parcelles présentent a priori des niveaux différents de fourniture de phosphore et de potassium par le sol. Par ailleurs, elles ont été soumises ou non à des apports d'engrais. La comparaison entre traitements, des biomasses aériennes produites et des teneurs de l'herbe en phosphore et en potassium, permet de définir des courbes de dilution de référence au-delà desquelles les prélèvements supplémentaires (ou les apports d'engrais) n'accroissent plus les quantités de biomasse produite. La validité des références établies antérieurement pour l'azote a été vérifiée. Les courbes de dilution de référence peuvent être utilisées pour porter un diagnostic sur l'état nutritionnel en $N, P$ et $K$ des prairies. Les cinétiques de dilution des différents éléments dans la biomasse étant similaires, les teneurs en $P$ et $K$ ont été exprimées en fonction de celles en $\mathrm{N}$, en accord avec des travaux antérieurs. Afin de faciliter le diagnostic, des indices de nutrition ont été définis en calculant le rapport entre la teneur observée et la teneur potentielle en l'élément considéré.
\end{abstract}

nutrition minérale / N/P/K/diagnostic / prairie

Summary - Diagnosis of nutrient level for permanent grasslands in spring. Building models. The pattern of nitrogen, phosphorus and potash uptake was studied in 4 permanent grasslands during the spring. These grasslands differed in their soil fertility level. Experimental treatments consisted of nitrogen (spread on the land each year at different places $\left.N_{1}=120 \mathrm{~kg}_{\mathrm{ha}}{ }^{-1}, \mathrm{~N}_{0}=0\right)$ ), phosphorus (52 kg.ha ${ }^{-1}$ or not) and potassium (208 kg.hat or not) spread on the land over a period of 4 years in the same place: Mineral uptake and mineral content were compared to aerial dry matter yield. Comparison between plots and treatments allowed us to define upper curves for which mineral content increased without dry matter yield increasing. Deviations from these curves were used to diagnose the herbage nutrition level. In order to simplify the method, the relationships between $N$ content and $P$ or $K$ content were analyzed. A slight deviation occurred with the second method in the case of nitrogen stress. A nutrient index has been described as the ratio between the observed mineral content and the potential content of the same mineral.

herbage nutrition / N/P/K/diagnosis/grass/and 


\section{INTRODUCTION}

Le calcul de la fumure minérale à apporter pour assurer une alimentation optimale des cultures s'appuie sur une estimation des besoins des plantes diminués des restitutions des précédents culturaux qu'on augmente éventuellement des pertes potentielles, ainsi que des immobilisations possibles au niveau du sol. Les quantités ainsi calculées sont ensuite majorées ou minorées en fonction du pouvoir alimentaire du sol. Les besoins sont généralement estimés à partir de l'établissement de courbes de réponse du rendement de la culture à des doses croissantes d'apport d'engrais. On en déduit alors les quantités contenues dans les parties récoltables au-delà desquelles le rendement n'augmente plus. L'estimation de l'offre alimentaire du sol requiert un diagnostic établi à partir de résultats de l'analyse de terre (tests de teneurs en minéraux "assimilables", $\mathrm{pH}$, texture, etc) et d'une hypothèse d'enracinement.

Le souhait de contrôler le niveau de nutrition, afin de vérifier le bien fondé des pratiques de fertilisation, a conduit à mettre au point des tests faisant appel à la teneur des plantes en éléments minéraux. Le plus souvent, il s'agit des feuilles. L'avantage de cette démarche est de ne pas reposer sur des hypothèses tant sur l'enracinement que sur l'assimilabilité des minéraux extraits chimiquement, comme c'est le cas pour l'analyse de terre courante. Outre le fait que chacune de ces méthodes possède des limites spécifiques, elles exigent la détermination de normes ou seuils de références qui doivent pouvoir s'appliquer dans des conditions de milieux et de techniques variées.

Les valeurs de référence des teneurs des plantes correspondent aux teneurs au-delà desquelles it n'y a plus d'augmentation correspondante de rendement. Les écarts par rapport à ces teneurs permettent d'évaluer les carences et les excès. Une difficulté d'utilisation tient au fait que les teneurs des plantes évoluent avec leur âge, ce qui oblige à fixer des teneurs de référence dépendant des stades de développement (phénologiques le plus souvent) bien précis (Martin et Matocha, 1972).
Pour s'affranchir des effets de l'âge, Beaufils (1973) a proposé de calculer des index de nutrition ( $\mathrm{N}, \mathrm{P}$ et $\mathrm{K}$ ) à partir de combinaisons des teneurs de chacun de ces éléments. Le diagnostic du niveau de nutrition d'une culture est basé sur la comparaison des valeurs des index obtenus pour une culture donnée aux index de référence en conditions de nutrition non limitante. Mais une limite sérieuse tient à l'existence d'importantes interactions qu'il est difficile de prendre en compte (Bates, 1971). C'est la raison pour laquelle le niveau de nutrition azotée sera pris en compte alors que notre objectif de départ est de nous limiter à la nutrition phosphopotassique.

Pour estimer le niveau de nutrition azotée de graminées fourragères en s'affranchissant des variations de teneur en fonction de l'âge, Salette et Lemaire (1981) ont proposé de modéliser la teneur en fonction de la biomasse aérienne récoltable. II a ainsi été montré l'existence d'une courbe limite (teneur en azote - rendement en matière sèche) au-delà de laquelle tout prélèvement supplémentaire d'azote se traduit par un accroissement de teneur sans augmentation de rendement. Ces courbes sont relativement similaires pour la plupart des graminées (Lemaire et Salette, 1984) et légumineuses comme la luzerne (Lemaire et al, 1985). La même approche a été réalisée pour les teneurs en $\mathrm{P}$ et $\mathrm{K}$ de graminées fourragères (Salette, 1982; Salette et al, 1982; Salette et Huché, 1989). Mais dans une perspective de simplification, ces auteurs ont proposé d'exprimer les teneurs de $\mathrm{P}$ et $\mathrm{K}$ en fonction de celle de l'azote dans la mesure où les courbes de dilution de ces minéraux sont relativement similaires.

Les cinétiques de prélèvement et de dilution des principaux minéraux $(N, P, K)$ ont été étudiées dans des prairies permanentes différenciées par le statut chimique du sol. Par ailleurs, ces prairies sont soumises ou non à une fertilisation phosphopotassique. Les comparaisons des cinétiques de dilution interprairie et intraprairie devrait nous permettre de déterminer des courbes limites de relation entre minéraux, ayant valeur de référence pour réaliser un diagnostic de nutrition. Lors de l'établissement de ces comparaisons, nous insisterons tout particulièrement sur les interactions entre éléments minéraux. 


\section{MATÉRIEL ET MÉTHODES}

\section{Site et protocole expérimental}

Quatre parcelles, situées à $1250 \mathrm{~m}$ dans les Pyrénées Centrales sur un sol de dépôts glaciaires, ont été échantillonnées d'après leur histoire culturale, facteur dont nous avons montré précédemment l'incidence sur la composition botanique et la productivité des prairies (Balent et Duru, 1984). Ces parcelles dont les principales caractéristiques sont présentées dans le tableau I (analyse de sol, composition botanique), sont nommées B (bon),
$M$ (moyen), C (carencé), $T$ (très pauvre), en fonc tion des quantités de biomasses récoltées en absence d'engrais. Deux dispositifs ont été mis en place.

Le dispositif témoin a pour objet de comparer les prélèvements et les teneurs en éléments minéraux de 4 parcelles différenciées par le statut chimique du sol. Il comprend des sous-ensembles de placettes mises en défens dès début novembre et des sous-ensembles de placettes pâturées par des ovins jusqu'à début mai. Les apports d'azote $(120 \mathrm{~kg} / \mathrm{ha})$ ont lieu début mars pour les placettes non mises en défens ou juste à l'arrêt du pâturage dans le second cas. Selon qu'il y ait ou non apport d'azote, la lettre caractérisant la parcelle est suivie

Tableau I. Principales caractéristiques des parcelles étudiées: analyse chimique de terre, composition botanique (nombre de contacts/100 pour les espèces dont le nombre de contacts par la méthode des points quadrats est supérieur à $50 \%$, d'après Balent, comm pers).

\begin{tabular}{|c|c|c|c|c|c|}
\hline & & $B$ & $M$ & $C$ & $T$ \\
\hline \multicolumn{6}{|c|}{ Analyse de terre } \\
\hline $\begin{array}{l}\mathrm{pH} \\
\mathrm{MO}(\%) \\
\mathrm{P}_{2} \mathrm{O}_{5}(\% 0) \\
\text { assimil } \\
\mathrm{K}_{2} \mathrm{O}(\% 0) \\
\text { échang. }\end{array}$ & $\begin{array}{c}0-15 \mathrm{~cm} \\
0-15 \mathrm{~cm} \\
0-5 \mathrm{~cm} \\
5-10 \mathrm{~cm} \\
10-15 \mathrm{~cm} \\
15-25 \mathrm{~cm} \\
25-40 \mathrm{~cm} \\
40-70 \mathrm{~cm} \\
0-5 \mathrm{~cm} \\
5-10 \mathrm{~cm} \\
10-15 \mathrm{~cm} \\
15-25 \mathrm{~cm} \\
25-40 \mathrm{~cm} \\
40-70 \mathrm{~cm}\end{array}$ & $\begin{array}{l}6,5 \\
7 \\
0,165 \\
0,072 \\
0,054 \\
0,044 \\
0,031 \\
0,050 \\
0,33 \\
0,15 \\
0,11 \\
0,10 \\
0,06 \\
0,06\end{array}$ & $\begin{array}{l}6,7 \\
5,5 \\
0,052 \\
0,039 \\
0,037 \\
0,045 \\
0,046 \\
0,057 \\
0,19 \\
0,19 \\
0,08 \\
0,06 \\
0,04 \\
0,03\end{array}$ & $\begin{array}{l}6,3 \\
10 \\
0,052 \\
0,025 \\
0,021 \\
0,027 \\
0,011 \\
0,023 \\
0,17 \\
0,17 \\
0,06 \\
0,05 \\
0,04 \\
0,03\end{array}$ & $\begin{array}{l}5,7 \\
5,7 \\
0,066 \\
0,035 \\
0,037 \\
0,029 \\
1 \\
1 \\
0,42 \\
0,42 \\
0,31 \\
0,21 \\
1 \\
1\end{array}$ \\
\hline $\begin{array}{c}\text { Composition } \\
\text { Dactylis glc } \\
\text { Cynosurus } \\
\text { Festuca ru } \\
\text { Lolium pert } \\
\text { Poa trivialis } \\
\text { Agrostis ca } \\
\text { Trisetum fla } \\
\text { Arrhenathe } \\
\text { Holcus lan } \\
\text { Anthoxanth }\end{array}$ & $\begin{array}{l}\text { otanique } \\
\text { merata } \\
\text { cristatus } \\
\text { ra } \\
\text { nne } \\
\text { illaris } \\
\text { vescens } \\
\text { um elatius } \\
\text { tus } \\
\text { im odoratum }\end{array}$ & $\begin{array}{c}70 \\
1 \\
1 \\
78 \\
58 \\
1 \\
1 \\
1 \\
1 \\
1\end{array}$ & $\begin{array}{c}68 \\
68 \\
1 \\
1 \\
1 \\
52 \\
1 \\
1 \\
1 \\
68\end{array}$ & $\begin{array}{c}1 \\
1 \\
56 \\
1 \\
54 \\
66 \\
58 \\
54 \\
1 \\
1\end{array}$ & $\begin{array}{c}1 \\
1 \\
94 \\
1 \\
1 \\
1 \\
1 \\
1 \\
1 \\
1\end{array}$ \\
\hline $\begin{array}{l}\text { Trifolium re } \\
\text { Trifolium pr } \\
\text { Trifolium ca } \\
\text { Lotus corni }\end{array}$ & $\begin{array}{l}\text { ens } \\
\text { atense } \\
\text { mpestre } \\
\text { iulatus }\end{array}$ & $\begin{array}{c}84 \\
1 \\
1 \\
1\end{array}$ & $\begin{array}{c}62 \\
1 \\
1 \\
1\end{array}$ & $\begin{array}{c}1 \\
58 \\
1 \\
60\end{array}$ & $\begin{array}{c}1 \\
1 \\
58 \\
1\end{array}$ \\
\hline $\begin{array}{l}\text { Taraxacum } \\
\text { Plantago m } \\
\text { Sanguisorb } \\
\text { Ranunculus } \\
\text { Cerastium } \\
\text { Rhinantus }\end{array}$ & $\begin{array}{l}\text { officinalis } \\
\text { ajor } \\
\text { minor } \\
\text { acris } \\
\text { ontanum } \\
p\end{array}$ & $\begin{array}{c}62 \\
58 \\
/ \\
1 \\
1 \\
1\end{array}$ & $\begin{array}{l}1 \\
68 \\
1 \\
56 \\
64 \\
58\end{array}$ & $\begin{array}{c}1 \\
1 \\
76 \\
1 \\
1 \\
1\end{array}$ & $\begin{array}{c}1 \\
1 \\
58 \\
1 \\
1 \\
1\end{array}$ \\
\hline
\end{tabular}


du chiffre 0 ou 1. Pour être certain de disposer d'une situation sans limitation de nutrition $P$ et $K$, les placettes de la prairie $B$ ont reçu des apports annuels de $120 \mathrm{~kg}$ de $\mathrm{P}_{2} \mathrm{O}_{5}$ et $250 \mathrm{~kg}$ de $\mathrm{K}_{2} \mathrm{O}$ (tableau II).

Le deuxième dispositif a comme but d'étudier les effets de la fertilisation $P$ et $K$ pour les prairies $M, C$ et $T$ présentant à priori une déficience en ces éléments. Trois modalités de fertilisation ont été appliquées sur ces parcelles (tableau II). L'incidence des apports de $\mathrm{K}(250 \mathrm{~kg} \mathrm{~K} \mathrm{O} / \mathrm{ha})$, a été étudiée en 1987 et 1988 mais seulement sur les repousses faisant suite à un pâturage et par des ovins de début novembre à début mai. Les apports conjoints de $\mathrm{P}$ et $\mathrm{K}$ ont concerné des repousses après pâturage sans apport d'azote en 1986 et 1987, mais sans pâturage et avec apport d'azote $(120 \mathrm{~kg} / \mathrm{ha})$ en 1988 . Les fertilisations $P$ et $K$ de ce dispositif sont apportées annuellement sur les mêmes emplacements depuis 1985 (apports conjoints de $\mathrm{P}$ et $\mathrm{K}$ ), ou 1987 (apport de K seul).

\section{Mesures}

Six à sept prélèvements d'herbe ont été réalisés annuellement de début avril à début juillet pour les sous-parcelles non pâturées au printemps et 4 pour les sous-parcelles pâturées. Les prélèvements ont été réalisés sur des placettes de $0,25 \mathrm{~m}^{2}$ avec 4 répétitions pour chaque traitement. Les coupes successives, effectuées à $2 \mathrm{~cm}$ de hauteur environ, ont eu lieu à des emplacements différents d'une même parcelle. Après séchage puis pesée, les échantillons d'herbe des 4 répétitions ont été mélangés pour l'analyse minérale.

Ce double dispositif a permis d'étudier les relations entre les teneurs des plantes et les biomasses aériennes, d'une part pour un ensemble de parcelles dont les sols présentaient à priori un gradient de fertilité $P$ et $K$, et d'autre part pour ces mêmes parcelles après une fertilisation minérale.

\section{RÉSULTATS}

\section{Quantités d'éléments minéraux contenus dans la biomasse aérienne récoltée}

Les résultats de 1986 du premier dispositif sont représentés à titre d'exemple sur la figure 1. Les quantités prélevées les plus faibles sont observées pour les parcelles $T$ (azote et phosphore) et C (potassium). Elles sont toujours inférieures pour les parcelles n'ayant pas reçu d'azote. Pour les traitements $\mathrm{N}_{1}$, on note à partir de la $3^{\mathrm{e}}$ date de prélèvement (20 mai) une réduction de la quantité d'éléments prélevés pour la parcelle $B$ et un ralentissement ou une stabilisation pour les parcelles $M$ et $C$. Pour les traitements $N_{0}$, on observe les mêmes phénomènes mais seulement à partir de la $4^{\mathrm{e}}$ ou $5^{\mathrm{e}}$ date de contrôle. Les mêmes observations ont été faites en 1987 et 1988.

D'une manière générale, les absorptions cessent à partir du début de l'épiaison pour les talles reproductrices de graminées fourragères (Williams, 1957). Dans notre situation, l'épiaison du dactyle a lieu à la première décade du mois de juin (Duru et Gibon, 1989).

Tableau II. Parcelles et traitements expérimentaux étudiés selon les années : (6-7-8 correspondant à 1986, 1987, 1988); $\bar{D}$ parcelles non défoliées en hiver, $D$ parcelles défoliées en hiver, $\left({ }^{\star}\right)$ apport annuel pour la prairie B, apport cumulé chaque année sur les mêmes emplacements depuis 1985 (prairies $M$ et $C$ ) ou 1986 (prairie T). L'apport ou non d'engrais est indiqué respectivement par les indices 1 et 0 pour chaque élément.

\begin{tabular}{|c|c|c|c|c|c|c|c|c|}
\hline \multirow[b]{3}{*}{ Défoliation hivernale } & \multicolumn{8}{|c|}{ Parcelles } \\
\hline & \multicolumn{2}{|c|}{$B$} & \multicolumn{2}{|c|}{$M$} & \multicolumn{2}{|c|}{$C$} & \multicolumn{2}{|c|}{$T$} \\
\hline & $\bar{D}$ & $D$ & $\bar{D}$ & $D$ & $\bar{D}$ & $D$ & $\bar{D}$ & $D$ \\
\hline $\begin{array}{lll}P_{0} & K_{0} & N_{1} \\
P_{0} & K_{0} & N_{0}\end{array}$ & & & $\begin{array}{l}6-7-8 \\
6-7-8\end{array}$ & & & & $\begin{array}{l}6-7-8 \\
6-7-8\end{array}$ & \\
\hline $\mathrm{P}_{1} \mathrm{~K}_{1} \mathrm{~N}_{1}\left(^{*}\right)$ & $6-7-8$ & & 8 & $6-7-8$ & 8 & $6-7-8$ & 8 & $6-7-8$ \\
\hline $\mathrm{P}_{1} \mathrm{~K}_{1} \mathrm{~N}_{0}\left(^{*}\right)$ & & & & $6-7-8$ & & $6-7-8$ & & $6-7-8$ \\
\hline$P_{0} K_{1} N_{1}$ & & & & $7-8$ & & $7-8$ & & $7-8$ \\
\hline
\end{tabular}



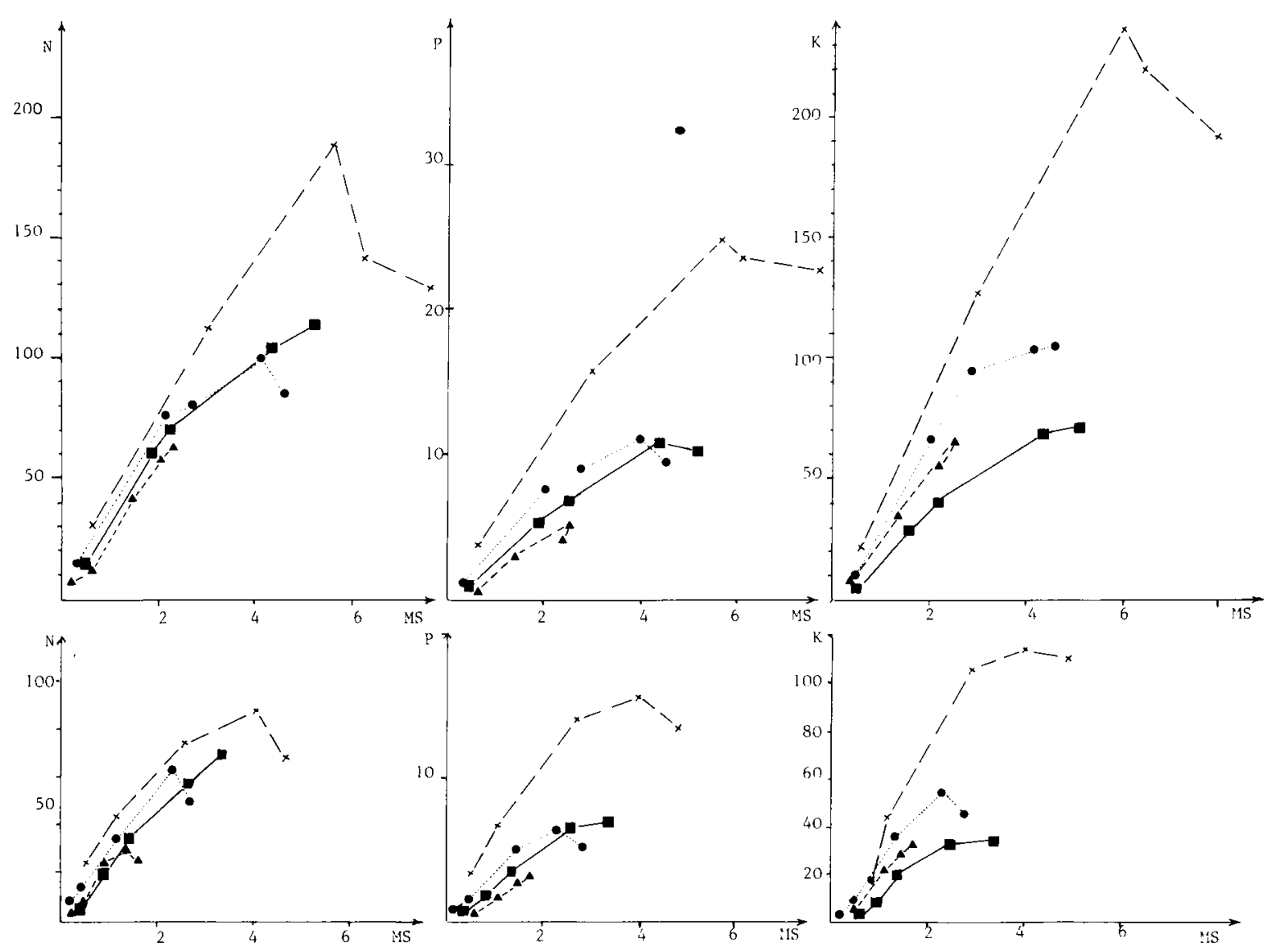

Fig 1. Prélèvement net de quantités de minéraux $N, P$ et $K\left(\mathrm{~kg} \cdot \mathrm{ha}^{-1}\right)$ en fonction de la biomasse récoltée (MS en $\left.t \cdot h a^{-1}\right)$ lors de la repousse de printemps en 1986. Partie haute des figures: $N=120 \mathrm{~kg}^{\mathrm{h}} \mathrm{h}^{-1}$, partie basse : sans azote. Parcelles $\mathrm{x}: \mathrm{B}, \boldsymbol{Q}: \mathrm{M}, \mathbf{\mathrm { a }}: \mathrm{C}, \mathbf{\Delta}: \mathrm{T}$.

\section{Dilution des éléments minéraux}

Les cinétiques de dilution des éléments minéraux en fonction de la biomasse produite sont représentées pour les parcelles $B$ et $C$ du premier dispositif en 1986 avec ou sans apport d'azote (fig 2).

\section{Azote (fig 2a)}

On note que les teneurs des échantillons de la placette $B_{1}$ sont supérieures à celles de la courbe de référence établie par Lemaire et al (1989), sauf à partir de début juin en liaison avec l'apparition d'une sénescence importante. Les teneurs de l'herbe de la placette $\mathrm{C}_{1}$ sont, par contre, nettement inférieures à celles de la placette $B_{1}$ alors que les apports d'azote sont les mêmes. À chaque date, on note des différences de teneur et de biomasse, ce qui laisse supposer une carence en azote dans le cas de la placette $C_{1}$.

\section{Phosphore (fig 2b)}

Les courbes sont similaires à celles de l'azote, mais les différences en fonction des apports d'azote sont plus faibles. Les 2 parcelles $B$ et $C$ sont dans ce cas très nettement individualisées. L'apport d'azote se traduit par un accroissement des teneurs en $\mathrm{P}$ pour une même biomasse récoltée.

\section{Potassium (fig 2c)}

On observe d'abord une certaine constance, voire un léger accroissement des teneurs, puis une diminution. Les effets de l'azote sont similaires à ceux observés pour le phosphore.

Sur la figure 3 , nous avons représenté les dilutions de minéraux observées en 1988 pour les placettes $N_{1}$ après ou non apport de fertilisation $P$ et $K$ durant 4 années consécutives (comparaison des parcelles $\mathrm{M}, \mathrm{C}$ et $\mathrm{T}$ du premier et du deuxième dispositif) ou une année 


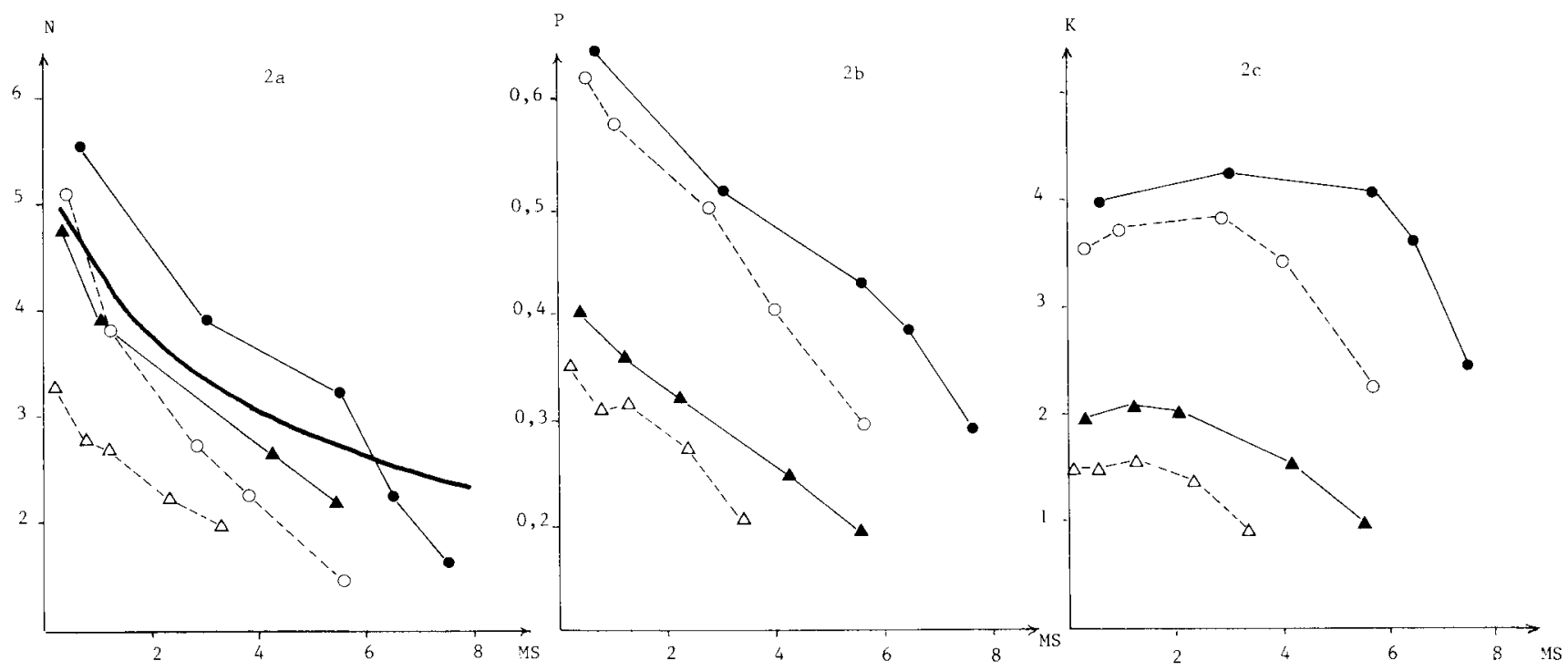

Fig 2. Teneurs en azote (2a), phosphore (2b) et potassium (2c) en pourcentage de la matière sèche en fonction de la biomasse récoltée en $1986\left(\mathrm{t} \cdot \mathrm{ha}^{-1}\right)$ pour les parcelles $\mathrm{B}(\boldsymbol{\bullet}, O)$ et $\mathrm{C}(\boldsymbol{\Lambda}, \Delta)$ avec azote $(\mathbf{\Lambda}, \mathbf{\Delta})$ et sans azote $(O, \Delta)$ courbe de référence $\mathrm{N}$ non limitant (Lemaire et al, 1989)
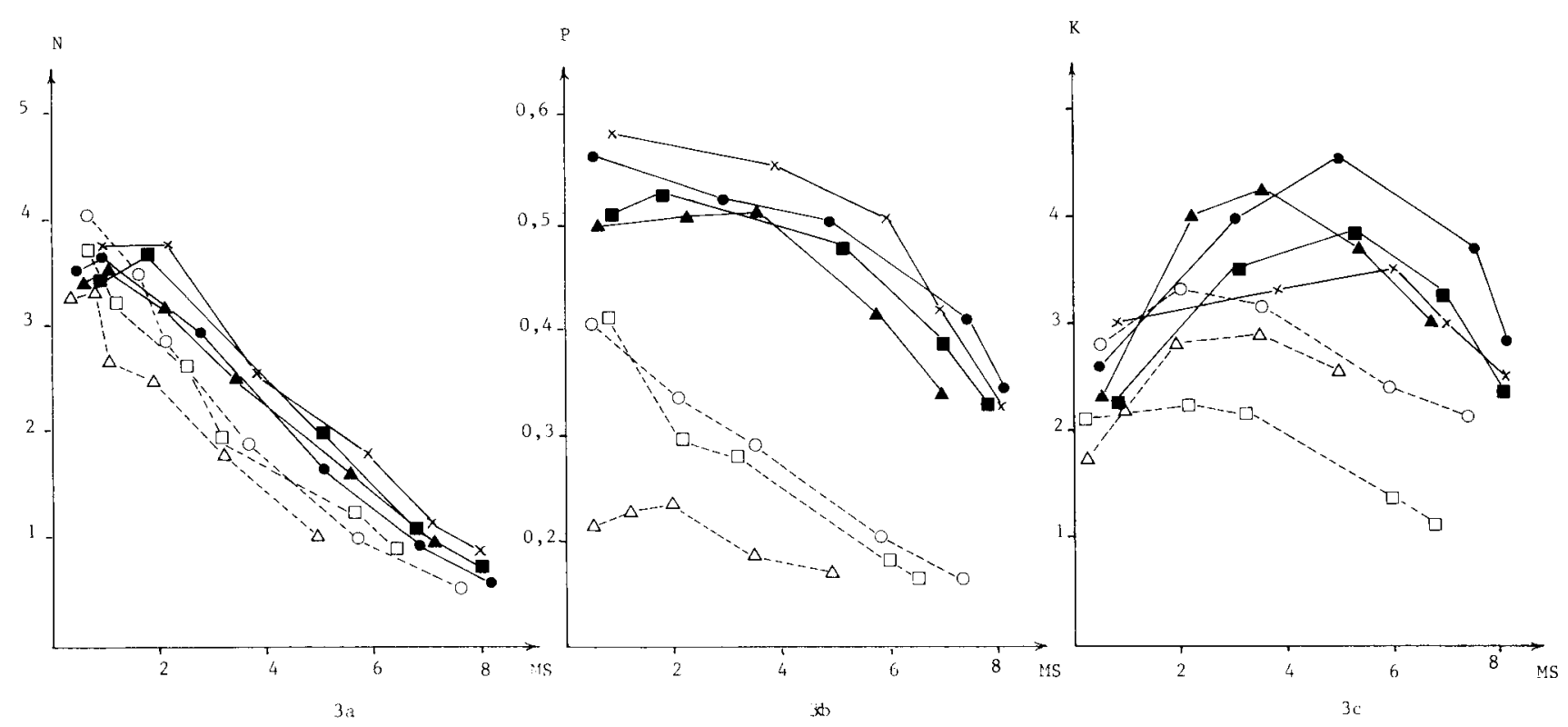

Fig 3. Dilution de l'azote (3a), du phosphore (3b) et du potassium (3c), (\%MS) en fonction de la biomasse aérienne récoltée en 1988 (t.ha ${ }^{-1}$ ) pour les parcelles $B(x), M(\bullet, O), C(\boldsymbol{\square}, \square)$ et $T(\mathbf{A}, \Delta)$ ayant reçu de l'azote. Les symboles noirs correspondent aux placettes avec apports de $P$ et $K$ cumulés durant 4 années.

(parcelle B). Après fertilisation PK, on observe des courbes de dilution tout à fait voisines entre traitements et parcelles pour $\mathrm{N}$ et le $\mathrm{P}$ et dans une moindre mesure pour $\mathrm{K}$. Par contre, pour les parcelles non fertilisées, les teneurs en $\mathrm{P}$ et $\mathrm{K}$, à biomasse donnée, sont très nettement inférieures pour le $P$ de toutes les parcelles et pour le $\mathrm{K}$ dans le cas de la parcelle $\mathrm{C}$.

\section{Relations entre les teneurs en éléments minéraux}

\section{Premier dispositif}

Les relations présentées sont celles des parcelles B et C avec apport d'azote (fig 4). 


\section{Phosphore}

Les courbes sont bien regroupées entre années et sont quasi linéaires avec une tendance asymptotique pour les teneurs les plus élevées. Celles de la parcelle $C$ se distinguent nettement de celles de la parcelle B (fig 4a).

\section{Potassium (fig 4b)}

Au cours de la croissance, on note souvent une phase où la teneur est stable ou s'accroît alors que celle en $\mathrm{N}$ diminue. Ensuite, les teneurs des 2 minéraux diminuent de manière

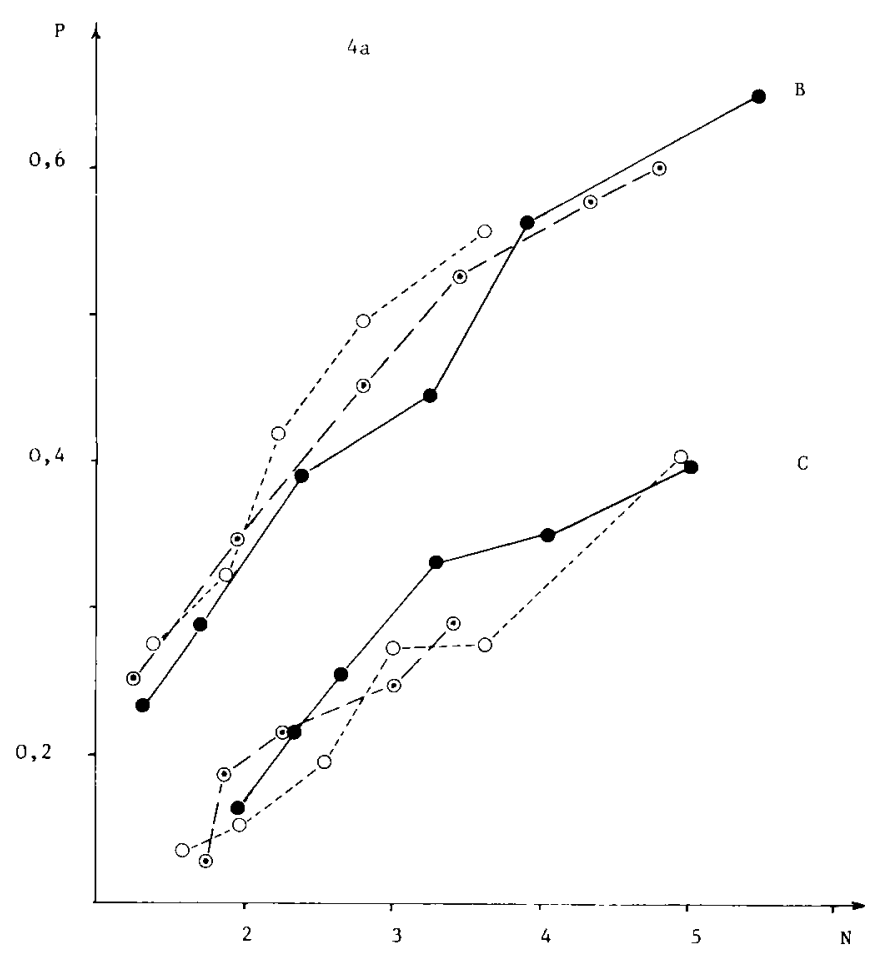

concomitante. Si seules sont retenues les données où la biomasse aérienne est $\geq 1,5 t /$ ha, les teneurs en $K$ sont toujours décroissantes. Cependant, les courbes représentatives des teneurs pour chaque année sont moins bien regroupées que pour le phosphore.

Étant donné que les relations $N \%, P \%$, $K \%=f(M S)$ sont du type $\alpha M S^{\beta}$, les relations entre minéraux ont été ajustées avec une fonction de même type. Les coefficients des relations pour l'ensemble des parcelles et les 2 traitements azote sont indiqués dans le tableau III.

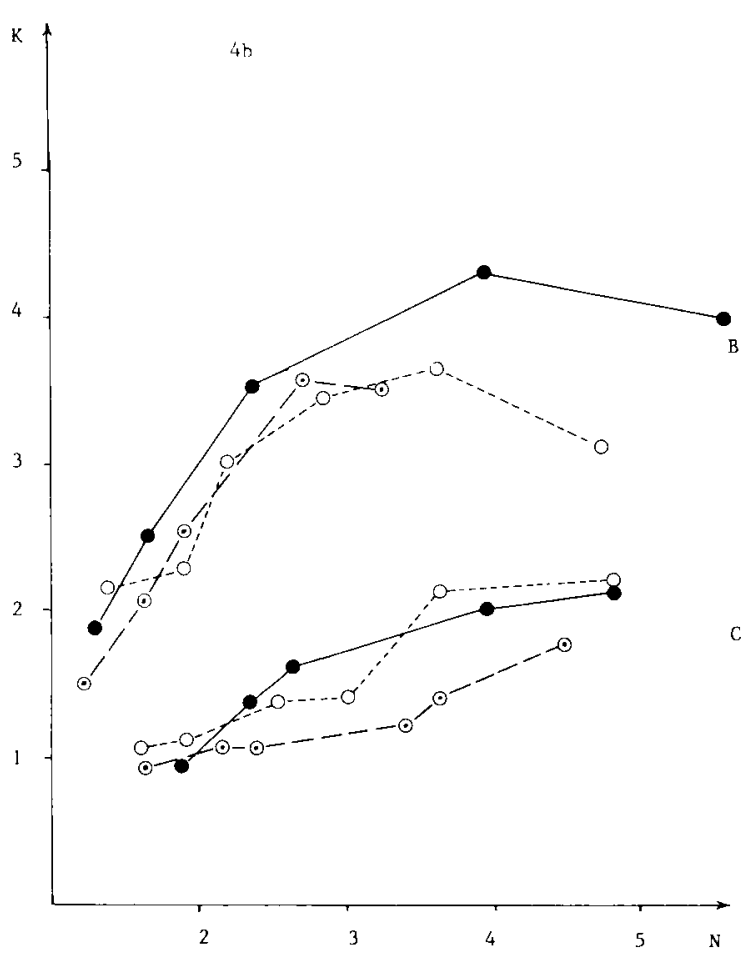

Fig 4. Relation entre teneur de $P$ et $N(4 a)$ d'une part et $K$ et $N$ (4b) d'autre part dans la biomasse aérienne pour les parcelles $B$ et $C$ en l'absence d'apport de P et K. $1986(\mathbf{0}), 1987(\odot)$ et $1988(\bigcirc)$.

Tableau III. Coefficients des relations $P(\%)$ ou $K(\%)=\alpha N^{\beta}$ pour les parcelles $B, M, C$ et $T$ avec azote $\left(N_{1}\right)$ et sans azote $\left(N_{0}\right)$ pour les données où $M S>1,5 \mathrm{t}$ dans le cas du potassium. Tous les coefficients de corrélation sont significatifs au seuil 0,001 sauf ${ }^{*}(0,01)$ et ${ }^{* *}(0,05)$.

\begin{tabular}{lcccccccc}
\hline & \multicolumn{9}{c}{$N_{1}$} & \multicolumn{5}{c}{$N_{0}$} \\
\cline { 2 - 7 } & $B$ & $M$ & $C$ & $T$ & $B$ & $M$ & $C$ & $T$ \\
\hline$P$ & & & & & & & & \\
$\alpha$ & 0,22 & 0,12 & 0,096 & 0,080 & 0,26 & 0,15 & 0,10 & 0,098 \\
$\beta$ & 0,68 & 0,79 & 0,92 & 0,91 & 0,59 & 0,77 & 0,94 & 0,91 \\
$n$ & 18 & 18 & 18 & 17 & 18 & 18 & 17 & 16 \\
$r$ & 0,97 & 0,97 & 0,96 & 0,96 & 0,94 & 0,90 & 0,91 & 0,95 \\
$K$ & & & & & & & & \\
$\alpha$ & 1,62 & 1,63 & 0,70 & 1,60 & 1,82 & 1,80 & 0,72 & 1,45 \\
$\beta$ & 0,75 & 0,54 & 0,71 & 0,42 & 0,71 & 0,43 & 0,70 & 0,58 \\
$n$ & 15 & 15 & 15 & 13 & 14 & 13 & 12 & 10 \\
$r$ & 0,88 & 0,94 & 0,81 & $0,62^{* *}$ & 0,88 & $0,75^{*}$ & $0,77^{*}$ & $0,78^{*}$ \\
\hline
\end{tabular}


Pour $K, \alpha$ décroît de $B$ en T alors que $\beta$ augmente. Ceci indique que les écarts entre parcelles sont les plus élevés en début de repousse mais qu'ils se réduisent par la suite. En absence d'apport d'azote, les coefficients $\alpha$ sont plus élevés mais les coefficients $\beta$ sont inférieurs ou égaux. II en résulte que les courbes $P \%-N \%$ auront une ordonnée à l'origine légèrement inférieure en cas d'apport d'azote. Ce constat découle des effets de l'azote sur les variations de teneur de chacun des minéraux présentés précédemment. Pour $\mathrm{K}$, seule la parcelle C se différencie nettement (tableau III). La plupart des coefficients de corrélation sont hautement significatifs, ce qui témoigne d'une bonne stabilité des données entre années.

Afin de tester la sensibilité de ces relations aux modes d'exploitations, nous les avons établies pour des repousses après pâturage. Sur la figure 5 , sont indiquées les données de la parcelle $M$. On note une bonne adéquation avec les courbes obtenues précédemment pour les parcelles non défoliées. Les valeurs calculées des paramètres dépendent peu des modalités d'exploitation. Elles peuvent donc être considérées comme caractéristiques d'une parcelle donnée.

\section{Deuxième dispositif}

Afin de comparer entre années les paramètres des courbes $P \%=f(N \%)$ et $K \%=f(N \%)$, nous utiliserons d'une part les données des repousses après pâturage pour K (1987, 1988 ) et pour $P$ (1985 à 1987), et d'autre part les données des placettes non défoliées pour l'étude des teneurs en P (1988).

Les apports de $K$, n'ont été réalisés que 2 années consécutives et en l'absence d'apport de P. La comparaison des différentes courbes de la figure 6 , montre que dans ce cas, ils n'ont pas eu d'effet sur l'ordonnée à l'origine saui pour la parcelle $\mathrm{C}$. Mais même après ces 2 années d'apports, les teneurs observées en $C$ sont inférieures à celles des parcelles $M$ et $T$.

Pour $P$, les résultats sont présentés figure 7. Dans tous les cas, il s'agit de parcelles azotées ayant reçu un apport simultané de $K$, donc sans facteur limitant autre que $P$. On note que les écarts des teneurs de $P$ entre placettes fertilisées ou non sont importants dès la première année (fig $7 \mathrm{a}$ ) et qu'ils s'amplifient les années suivantes (fig $7 \mathrm{~b}, \mathrm{c}$ ). En quatrième année, les courbes relatives aux 3 parcelles ayant bénéficié d'apports continus de $\mathrm{P}$ et $\mathrm{K}$, sont alors relativement voisines en-
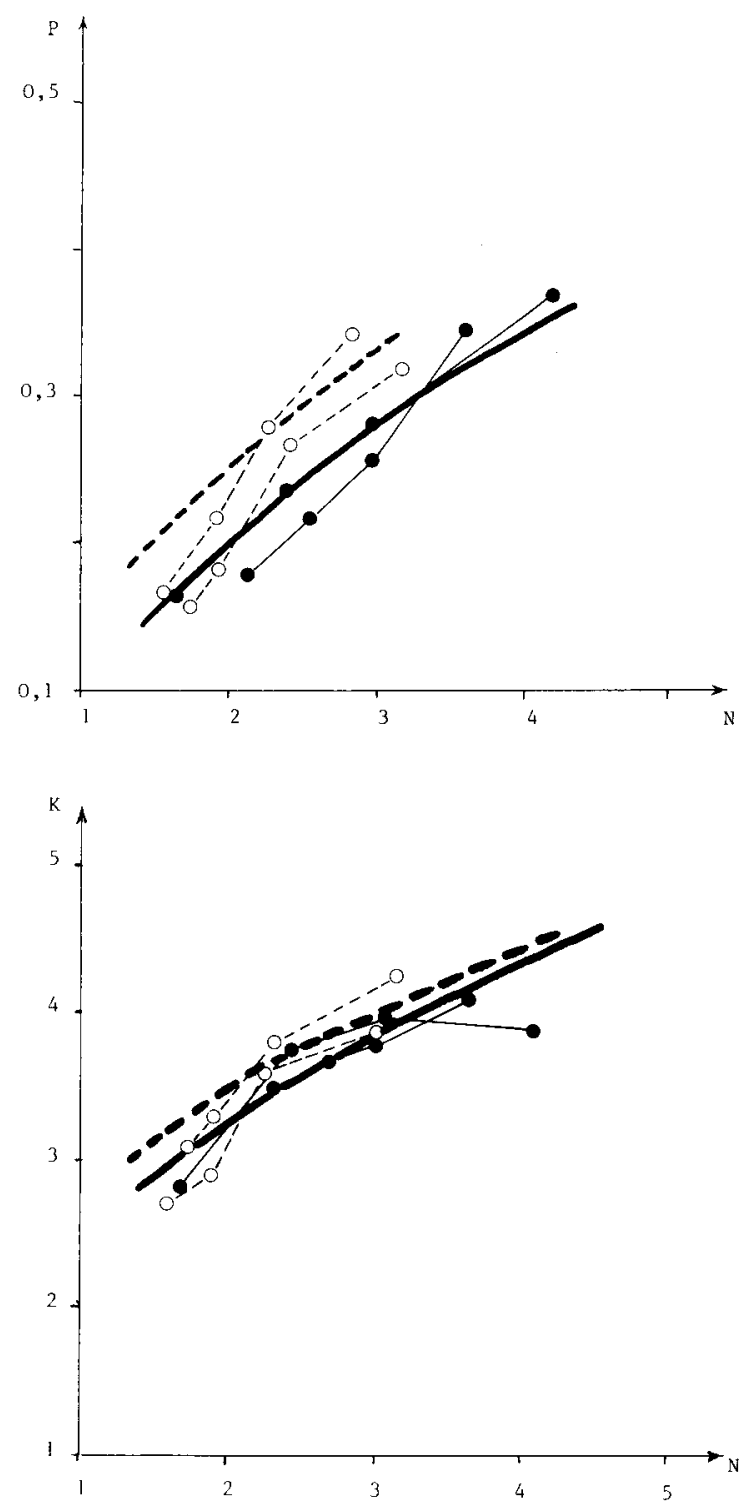

Fig 5. Comparaisons des relations entre teneurs en $P$ et $N(5 a), K$ et $N(5 b)$ de la biomasse aérienne pour les repousses pâturées en hiver et au début du printemps en $1986(0)$ et 1987 (@) de la parcelle M (-0 : avec azote, $\bigcirc---O$ : sans azote), aux ajustements obtenus pour la même parcelle non páturée (- avec azote, --- sans azote).

tre elles et de celle de la parcelle $B$. Les teneurs des parcelles $M, C$ et $T$ ont alors environ doublé (fig 7d).

\section{Effet des apports de $P$ et $K$ sur la production de matière sèche}

Nous ne considérerons que les placettes ayant reçu une fertilisation azotée. Pour chaque parcelle, nous comparons les biomasses aériennes récoltées entre traitements témoins (premier dispositif) et placettes ayant reçu des 

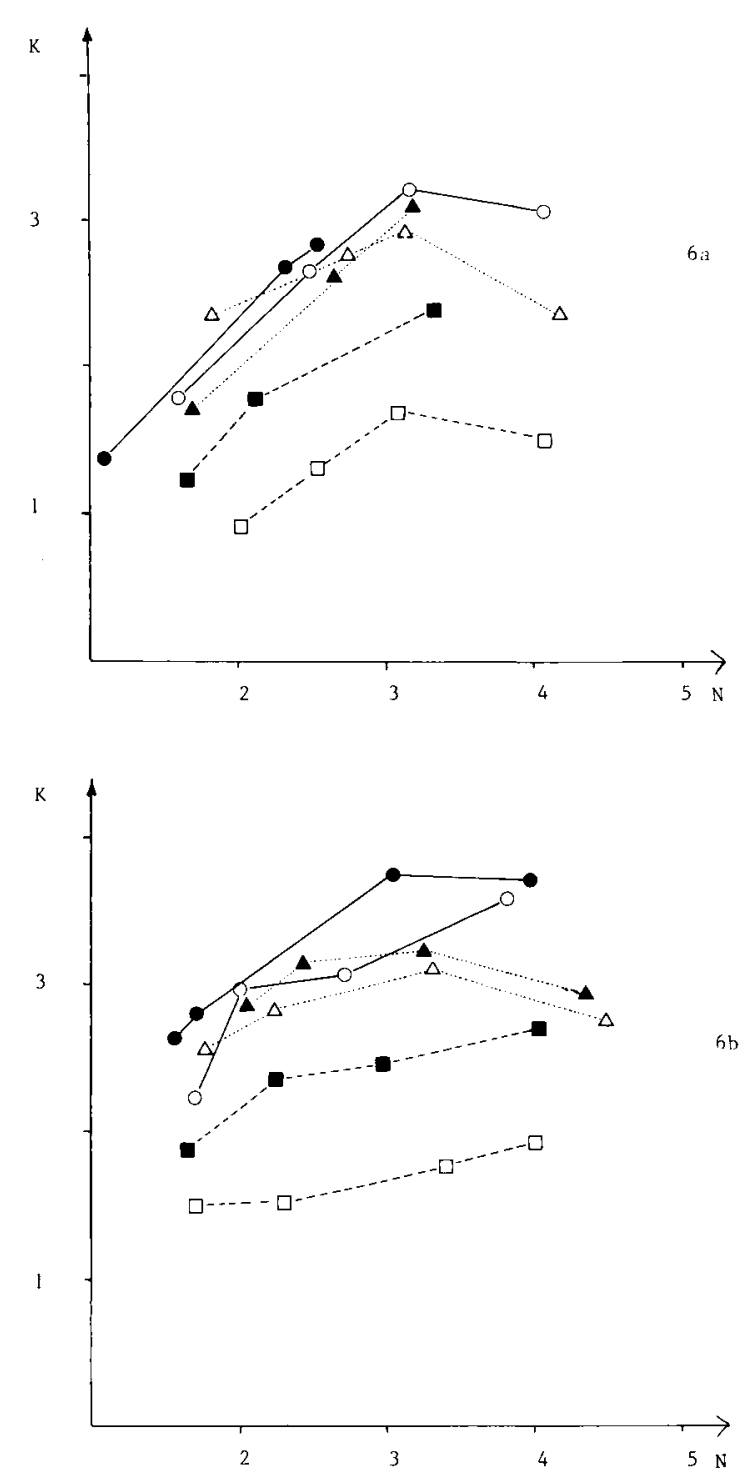

Fig 6. Relations entre les teneurs en $\mathrm{K}$ et $\mathrm{N}(\% \mathrm{MS})$ de la biomasse récoltée sur les parcelles ayant ou non reçu un apport de $K 1$ année (6a) ou 2 années (6b) consécutives. parcelles $M(O, 0), C(\square, \boldsymbol{D})$ et $T(\triangle, \boldsymbol{\Lambda})$ sans apports de $K$ (symboles blancs) et avec apports (symboles noirs).

apports d'engrais $P$ et $K$. En 1986 et 1987 $(P)$ et en $1988(K)$, les prélèvements ayant été réalisés sur des repousses après pâturage, nous ne diposons que des données à 4 dates pour comparer les traitements, ce qui est insuffisant pour établir les paramètres d'un modèle d'accumulation de biomasse en fonction du temps ou des températures. C'est pourquoi nous avons choisi de calculer date par date les différences moyennes de quantité de biomasse.

Les apports de $\mathrm{K}$ n'ont modifié les rendements de manière significative qu'en 1988 pour la parcelle $C$ (tableau IV). Pour $P$, on s'est affranchi des carences supposées des sols en $\mathrm{K}$ par apport systématique d'une fertilisation potassique. Nous notons qu'en présence d'azote, les accroissements de biomasse observés sont significatifs dès la première année de l'apport dans 1 cas sur 2 (tableau V). Ils sont significatifs les 3 années suivantes (sauf au dernier contrôle pour la parcelle T). Les accroissements moyens par parcelle varient de 12 à $59 \%$. Ils sont les plus importants pour la parcelle T. On note aussi que les effets moyens sont variables selon les années $+18 \%$ en $1985,+43 \%$ en $1986,+28 \%$ en $1987,+43 \%$ en 1988 .

\section{DISCUSSION}

\section{Absorption des éléments minéraux et conséquences sur leur dilution}

La comparaison des cinétiques de dilution des différents minéraux en fonction de la biomasse produite nécessite de rappeler les principaux processus dont dépend leur absorption. Pour $\mathrm{P}$ et $\mathrm{K}$, c'est l'approvisionnement en ions $\mathrm{PO}_{4}^{3-}$ et $\mathrm{K}^{+}$de la solution du sol où s'alimentent les racines, mais surtout l'exploration du sol par le système racinaire qui régissent l'absorption de ces éléments nutritifs. Mais les ions $\mathrm{PO}_{4}^{3-}$ et $\mathrm{K}^{+}$ne se déplacent dans le sol que sur de courtes distances, de l'ordre du $\mathrm{cm}$ pour $\mathrm{K}$ (Callot et al, 1982; Claasen et al, 1986), du $\mathrm{mm}$ pour $\mathrm{P}$ (Callot et al, 1982). Leur entraînement par mass flow y est très réduit; ce qui n'est pas le cas des ions nitriques, très mobiles, qui peuvent être transportés sur des distances bien plus importantes lorsque l'alimentation hydrique est non limitante (Calvet, 1988). Le rhyzocylindre est alors vite épuisé en $P$ et $K$, surtout dans les milieux où ces éléments sont peu disponibles. De ce fait, leur absorption dépend étroitement de la croissance racinaire, tout particulièrement pour $K$. C'est ainsi que Bosc et Maertens (1981) ont observé, pour la tomate, un parallélisme étroit entre les cinétiques de croissance racinaire et d'absorption des ions $\mathrm{K}$.

La comparaison de ces modes d'absorption nous amène à formuler l'hypothèse que la plus faible absorption de $\mathrm{K}$ et sa variabilité interannuelle plus importante que celle des autres minéraux au début du printemps peut 

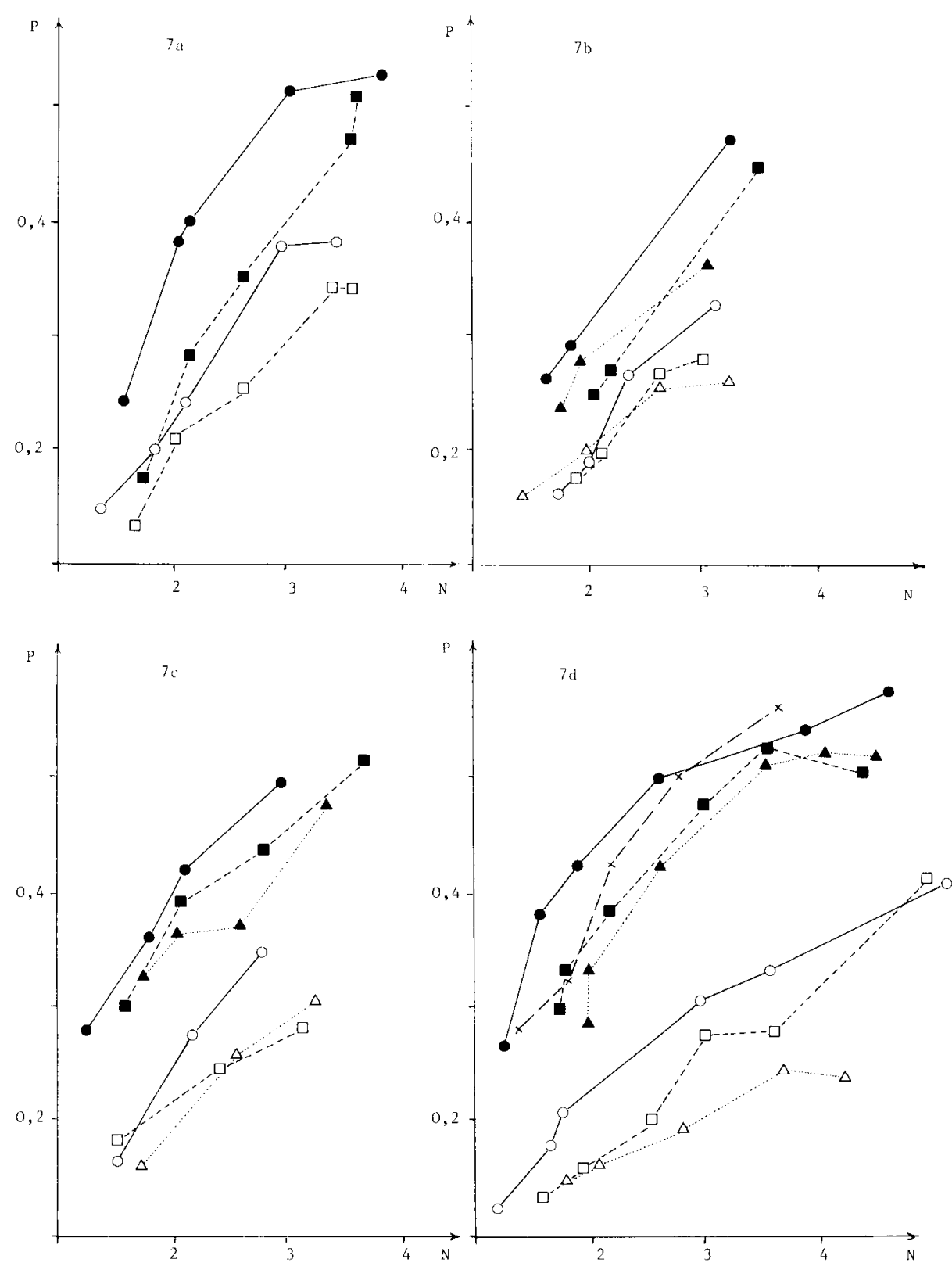

Fig 7. Relations entre les teneurs en $P$ et $N(\% M S)$ de la biomasse récoltée sur les parcelles fertilisées en $K$ et ayant ou non reçu un apport de $P$ et $K 1$ année (fig $7 \mathrm{a}) 2(7 \mathrm{~b}), 3(7 \mathrm{c})$ et 4 années consécutives (7d). Parcelles $M(O$, $)$; $C(\square, \boldsymbol{\square})$ et $T(\triangle, \boldsymbol{\Lambda})$ sans apport de $P$ (symboles blancs) et avec apport (symboles noirs).

être liée à un faible accroissement racinaire en relation avec les basses températures observées à cette période de l'année. La même observation a été faite pour les repousses après pâturage, mais dans ce cas, les faibles températures ne peuvent expliquer le résultat puisque le même rendement est obtenu un mois plus tard environ. Cependant, la même hypothèse peut être formulée, car il a été montré que la défoliation a pour effet d'interrompre momentanément la croissance racinaire (Gillet, 1980). Ces observations tendent donc à confirmer le rôle de la croissance ra- cinaire dans la dynamique d'absorption de $\mathrm{K}$ et à exclure, pour un diagnostic, les prélèvements récoltés trop tôt au printemps ou après une défoliation.

\section{Effet de la fertilisation} phosphopotassique sur le niveau de nutrition azotée

La différence de niveau de nutrition azotée observée après apport d'azote minéral entre 
Tableau IV. Biomasse récoltée $\left(\mathrm{g} \cdot \mathrm{m}^{-2}\right)$ en 1988 à différentes dates $(d i)$ pour les parcelles $\mathrm{M}, \mathrm{C}$ et $\mathrm{T}$ sans apport de $P$ mais avec apport de $K(1)$ ou non (0). À une date donnée, les résultats d'une même parcelle suivis d'une lettre différente sont significativement différents au seuil $5 \%$ (test de Newman-Keuls); moyenne et écart type des différences de biomasse (MS) par parcelle: $\left(M S_{1}-M S_{0}\right) / M S_{1} \times 100$.

\begin{tabular}{ccccccc}
\hline Parcelle & $\begin{array}{c}\text { Apport de } \\
K_{2} O(\mathrm{~kg} / \mathrm{ha})\end{array}$ & $d_{3}$ & $d_{4}$ & $d_{5}$ & $d_{6}$ & $\begin{array}{c}\text { Moyenne et } \\
\text { écart type }\end{array}$ \\
\hline $\mathrm{M}$ & 250 & $115^{\mathrm{a}}$ & $452^{\mathrm{a}}$ & $823^{\mathrm{a}}$ & $802^{\mathrm{a}}$ & $\mathrm{NS}$ \\
& 0 & $129^{\mathrm{a}}$ & $466^{\mathrm{a}}$ & $887^{\mathrm{a}}$ & $811^{\mathrm{a}}$ & \\
$\mathrm{C}$ & 250 & $99^{\mathrm{a}}$ & $380^{\mathrm{a}}$ & $554^{\mathrm{a}}$ & $624^{\mathrm{a}}$ & $19 \pm 14$ \\
& 0 & $69^{\mathrm{b}}$ & $340^{\mathrm{b}}$ & $492^{\mathrm{b}}$ & $442^{\mathrm{b}}$ & NS \\
$\mathrm{T}$ & 250 & $65^{\mathrm{a}}$ & $302^{\mathrm{a}}$ & $615^{\mathrm{a}}$ & $653^{\mathrm{a}}$ & $596^{\mathrm{b}}$ \\
\hline
\end{tabular}

Tableau V. Biomasse récoltée $\left(\mathrm{g} \cdot \mathrm{m}^{-2}\right.$ ) à différentes dates (di) pour les parcelles $M, C$ et $T$ avec apport de $\mathrm{N}$ et $\mathrm{K}$ mais avec apport de $\mathrm{P}(1)$ ou non $(0)$. À une date donnée, les résultats d'une même parcelle suivis d'une lettre différente sont significativement différents au seuil $5 \%$ (test de Newman-Keuls); moyenne et écart type des différences de biomasse (MS) par parcelle: $\left(M S_{1}-M S_{0}\right) / M S_{1} \times 100$.

\begin{tabular}{|c|c|c|c|c|c|c|c|c|}
\hline $\begin{array}{l}\text { Année } \\
\text { parcelle }\end{array}$ & $\begin{array}{c}\text { Apport de } \\
P_{2} \mathrm{O}_{5}(\mathrm{~kg} / \mathrm{ha})\end{array}$ & $d_{1}$ & $d_{2}$ & $d_{3}$ & $d_{4}$ & $d_{5}$ & $d_{6}$ & $\begin{array}{c}\text { Moyenne et } \\
\text { écart type }\end{array}$ \\
\hline \multicolumn{9}{|l|}{1985} \\
\hline $\mathrm{M}$ & $\begin{array}{r}120 \\
0\end{array}$ & & & $\begin{array}{l}29^{a} \\
24^{a}\end{array}$ & $\begin{array}{l}129^{\mathrm{a}} \\
117^{\mathrm{b}}\end{array}$ & $\begin{array}{l}261^{a} \\
261^{a}\end{array}$ & $\begin{array}{l}424^{a} \\
359^{b}\end{array}$ & $12 \pm 8$ \\
\hline$c$ & $\begin{array}{r}120 \\
0\end{array}$ & & & $\begin{array}{l}57^{\mathrm{a}} \\
48^{\mathrm{a}}\end{array}$ & $\begin{array}{l}182^{a} \\
149^{b}\end{array}$ & $\begin{array}{l}315^{a} \\
222^{b}\end{array}$ & $\begin{array}{l}451^{a} \\
384^{b}\end{array}$ & $25 \pm 10$ \\
\hline \multicolumn{9}{|l|}{1986} \\
\hline$M$ & $\begin{array}{r}120 \\
0\end{array}$ & & & $\begin{array}{l}168^{a} \\
115^{b}\end{array}$ & $\begin{array}{l}309^{\mathrm{a}} \\
200^{\mathrm{b}}\end{array}$ & $\begin{array}{l}397^{\mathrm{a}} \\
225^{\mathrm{b}}\end{array}$ & $\begin{array}{l}352^{a} \\
237^{b}\end{array}$ & $56 \pm 12$ \\
\hline$C$ & $\begin{array}{r}120 \\
0\end{array}$ & & & $\begin{array}{l}186^{a} \\
123^{b}\end{array}$ & $\begin{array}{l}292^{a} \\
198^{b}\end{array}$ & $\begin{array}{l}419^{a} \\
291^{b}\end{array}$ & $\begin{array}{l}416^{a} \\
316^{b}\end{array}$ & $43 \pm 7$ \\
\hline $\mathrm{T}$ & $\begin{array}{r}120 \\
0\end{array}$ & & & $\begin{array}{l}140^{a} \\
183^{b}\end{array}$ & $\begin{array}{l}222^{a} \\
164^{b}\end{array}$ & $\begin{array}{l}262^{a} \\
220^{b}\end{array}$ & $\begin{array}{l}241^{a} \\
252^{a}\end{array}$ & $31 \pm 25$ \\
\hline \multicolumn{9}{|l|}{1987} \\
\hline $\mathrm{M}$ & $\begin{array}{r}120 \\
0\end{array}$ & & & $\begin{array}{l}131^{\mathrm{a}} \\
110^{\mathrm{a}}\end{array}$ & $\begin{array}{l}314^{\mathrm{a}} \\
230^{\mathrm{b}}\end{array}$ & $\begin{array}{l}425^{a} \\
343^{b}\end{array}$ & $\begin{array}{l}529^{a} \\
470^{b}\end{array}$ & $23 \pm 9$ \\
\hline C & $\begin{array}{r}120 \\
0\end{array}$ & & & $\begin{array}{r}104^{\mathrm{a}} \\
83^{\mathrm{a}}\end{array}$ & $\begin{array}{l}242^{\mathrm{a}} \\
191^{\mathrm{b}}\end{array}$ & $\begin{array}{l}370^{a} \\
295^{b}\end{array}$ & $\begin{array}{l}550^{a} \\
433^{b}\end{array}$ & $26 \pm 1$ \\
\hline $\mathrm{T}$ & $\begin{array}{r}120 \\
0\end{array}$ & & & $\begin{array}{l}78^{a} \\
68^{b}\end{array}$ & $\begin{array}{l}248^{a} \\
158^{b}\end{array}$ & $\begin{array}{l}378^{a} \\
244^{b}\end{array}$ & $\begin{array}{l}534^{a} \\
466^{b}\end{array}$ & $35 \pm 20$ \\
\hline \multicolumn{9}{|l|}{1988} \\
\hline$M$ & $\begin{array}{r}120 \\
0\end{array}$ & $\begin{array}{l}55^{a} \\
10^{b}\end{array}$ & $\begin{array}{r}163^{b} \\
89^{b}\end{array}$ & $\begin{array}{l}272^{a} \\
206^{b}\end{array}$ & $\begin{array}{l}536^{\mathrm{a}} \\
352^{\mathrm{b}}\end{array}$ & $\begin{array}{l}774^{\mathrm{a}} \\
549^{\mathrm{b}}\end{array}$ & & $41 \pm 24$ \\
\hline C & $\begin{array}{r}120 \\
0\end{array}$ & $\begin{array}{l}78^{a} \\
64^{b}\end{array}$ & $\begin{array}{l}160^{b} \\
125^{b}\end{array}$ & $\begin{array}{l}281^{a} \\
258^{b}\end{array}$ & $\begin{array}{l}530^{a} \\
328^{b}\end{array}$ & $\begin{array}{l}635^{a} \\
428^{b}\end{array}$ & & $28 \pm 16$ \\
\hline $\mathrm{T}$ & $\begin{array}{r}120 \\
0\end{array}$ & $\begin{array}{l}55^{\mathrm{a}} \\
49^{\mathrm{a}}\end{array}$ & $\begin{array}{l}94^{a} \\
46^{b}\end{array}$ & $\begin{array}{l}231^{a} \\
110^{b}\end{array}$ & $\begin{array}{l}352^{a} \\
206^{b}\end{array}$ & $\begin{array}{l}416^{\mathrm{a}} \\
355^{\mathrm{b}}\end{array}$ & & $59 \pm 39$ \\
\hline
\end{tabular}

2 parcelles présentant a priori des offres alimentaires en $P$ et $K$ différentes (fig 2a), peut avoir 3 origines: une différence de fourniture d'azote par le sol (comme pourrait le laisser penser la comparaison des courbes des 2 parcelles sans apport d'azote), une différence de composition botanique (légumineuse notamment) ou bien encore, une interaction avec les autres éléments minéraux. La comparaison de la courbe de dilution de la parcelle $B$ à celles des autres parcelles ayant reçu une fertilisation PK durant 4 années 
(fig 3a) sans que la flore ait beaucoup changé (Duru et Balent, non publié), nous permet de privilégier cette dernière hypothèse. Sachant que l'amélioration de la nutrition en $P$ et $K$ peut favoriser l'accroissement du système racinaire (Callot et al, 1982), la disponibilité de l'azote peut s'en trouver augmentée. Mais on peut aussi invoquer un couplage de l'absorption des différents ions.

\section{Variations des teneurs de $P$ et de $K$ en fonction de la biomasse aérienne et de sa teneur en azote}

L'expression des teneurs de $P$ et de $K$ en fonction de la quantité de biomasse montre un effet dépressif de la carence en azote sur le niveau de nutrition $P$ et $K$. Mais, exprimées en fonction des teneurs en azote, elles sont souvent plus élevées pour le traitement sans apport d'azote. Dans la perspective d'utiliser ce second mode d'expression à des fins de diagnostic, il importe d'étudier le biais ainsi introduit.

Pour comparer ces 2 modes d'expression des teneurs en $P$ et $K$, nous avons choisi empiriquement un couple de valeurs de référence correspondant à la période de montaison des graminées $\left(M S=3 \mathrm{t} \cdot \mathrm{ha}^{-1}\right.$; $N=2,5 \% \mathrm{MS}$ ). Le calcul des corrélations entre couples de valeur ainsi définis montre une bonne correspondance entre ces 2 modes d'expression $\left(r^{2}=0,78, n=22\right.$ pour $\mathrm{P}$ et $r^{2}=0,80$, $n=22$ pour $\mathrm{K}$ ). L'analyse détaillée des couples de points avec et sans azote montre que les teneurs en $P$ des placettes $N_{0}$ sont supérieures aux teneurs des placettes $N_{1}$ (sauf 2 cas en 1988) quand $P$ est exprimé en fonction de la teneur en azote. L'inverse est observé quand $P$ est exprimé en fonction de la biomasse aérienne (fig 2b). Les teneurs en $\mathrm{K}$ exprimées en fonction de la biomasse aérienne sont toujours supérieures (ou égales) lorsqu'il y a apport d'azote. Par contre, lorsqu'elles sont exprimées en fonction de la teneur en azote, tous les cas de figure ont été observés. Ces observations expliquent que le coefficient de corrélation entre les 2 modes d'expression est plus élevé lorsqu'on réalise les ajustements séparément pour les traitements azotés. Par exemple, pour les repousses avec azote, nous avons alors $r^{2}=0,95$ pour $P$ et 0,87 pour $\mathrm{K}$.
Dans une perspective d'utilisation des teneurs des plantes comme outil de diagnostic, on peut penser qu'une surestimation des niveaux de nutrition $P$ et $K$ sera faite pour les parcelles sans apport d'azote lorsque les teneurs, notamment en $P$, sont exprimées en fonction des teneurs en N. Toutefois, on tendra à retenir ce premier mode d'expression, étant donné sa commodité d'emploi, puisqu'il n'est pas nécessaire de disposer de mesure de biomasse. Cependant, on pourra limiter cette distorsion en disposant d'une placette non limitante en $\mathrm{N}$ sur les parcelles où un diagnostic est à réaliser.

\section{Courbes de dilution de référence}

L'étude des variations des teneurs de $\mathrm{P}$ et de $K$ par rapport à celles en $N$ d'une part, et des variations conjointes de biomasse récoltable d'autre part, nous permet de proposer des courbes enveloppes caractéristiques d'un niveau de nutrition donné.

Les apports de $\mathrm{K}$ entraînent une augmentation de rendement uniquement pour la parcelle $\mathrm{C}$. II en résulte que l'ordonnée à l'origine des courbes K-N établies pour les parcelles $M$ et $T$ correspond à une valeur maximale qui garantit un niveau non limitant de nutrition en $\mathrm{K}$.

Les apports d'engrais $\mathrm{P}-\mathrm{K}$ nous permettent de porter un diagnostic sur la nutrition en $P$ de l'herbe récoltée sur les parcelles $M$ et $T$ dans la mesure où ces parcelles ne sont pas déficientes en $\mathrm{K}$. Des accroissements de biomasses importants ont été observés chaque année et pour chaque parcelle (tableau $\mathrm{V}$ ). II en résulte que la courbe limite permettant de diagnostiquer une déficience en $P$ est comprise entre celles établies pour les parcelles $M$ et $B$. À défaut de données plus précises, nous considérons la courbe de la parcelle B comme courbe limite supérieure.

Les courbes ainsi déterminées nécessiteraient d'être précisées par des expérimentations comportant différentes doses d'apport d'engrais sur chacune des parcelles pour mieux établir à partir de quelle valeur limite on peut caractériser une déficience. Néanmoins, nous avons montré que les références obtenues ont été établies par excès. Nous proposons d'utiliser de telles courbes de référence en considérant que le degré de déficience est proportionnel aux écarts entre les 
données obtenues dans la parcelle à juger et ces courbes.

La comparaison du classement des courbes de dilution pour le $\mathrm{P}$ et le $\mathrm{K}$ montre des désaccords avec les résultats d'analyse de terre indiqués dans le tableau I. Pour le $\mathrm{P}$, la courbe de dilution de la parcelle $\mathrm{T}$ a l'ordonnée à l'origine la plus basse alors que les teneurs du sol en $P$ assimilable sont plus élevées que celles des parcelles $M$ et $C$. Pour le $\mathrm{K}$, la courbe de dilution la plus basse est celle de la parcelle $C$ alors que les concentrations en $\mathrm{K}$ échangeable sont peu différentes de celles de la parcelle $T$.

Peu d'articles font référence à ces modes d'expression pour les prairies. Nous pouvons toutefois comparer nos résultats aux travaux initiés par Salette et al (1973) sur Digitaria pour le $\mathrm{K}$. La courbe limite proposée est très proche de celle des parcelles $M$ et $T$. Pour des graminées fourragères dans l'Ouest de la France, les courbes de teneurs en fonction de la biomasse établies par Salette et al, (1982), Salette et Huché $(1989,1991)$ et reprises par Pons et al (1989) sont, pour $P$ et $K$ comprises entre celles des parcelles $M$ et $B$. Ces courbes sont par ailleurs tout à fait voisines de celles proposées par de Montard, (1988) pour des prairies permanentes dans le Massif central.

Pour $\mathrm{N}$, étant donné le bon accord observé, avant sénescence importante, entre la courbe de dilution de référence de Lemaire et al, (1989): $N=4,8 M S^{-0,32}$ et nos données pluriannuelles de la parcelle $B$ (résultats non publiés) et compte tenu de sa généralité (Lemaire et al, 1990), nous avons retenu cette référence. Elle correspond aux teneurs potentielles en conditions de nutrition non limitantes.

\section{Établissement d'indices de nutrition}

Afin de comparer plus aisément les parcelles et traitements de fertilisation que par des courbes, nous avons défini des indices de nutrition.

\section{Azote}

L'indice est défini comme le rapport entre la teneur observée $\left(N_{o b s}\right)$ et la teneur potentielle $\left(N_{\text {pot }}\right)$. Cependant, ce calcul brut n'est pas sa- tisfaisant puisque l'observation d'un grand nombre de courbes de dilution (Lemaire et $\mathrm{Sa}$ lette, 1984), montre que les différences de teneur en azote entre courbes tendent à être constantes au cours de la repousse de telle sorte que le rapport varie avec l'accumulation de biomasse. Afin de s'affranchir de cette difficulté, nous proposons de corriger la teneur observée en simulant sa valeur pour $1 \mathrm{t}$ de matière sèche $\left(N_{\text {sim }}\right)$ avec l'hypothèse que les écarts de teneurs sont constants au cours de la repousse. Cette hypothèse sera testée dans un deuxième article en s'assurant que des variations d'indice au cours de la repousse traduisent bien des variations de nutrition. Sous cette condition, nous avons

$$
\begin{gathered}
\left(N_{\text {pot }}-N_{\text {obs }}\right)=\left(4,8-N_{\text {sim }}\right), \\
N_{\text {sim }}=\left(4,8-4,8 M S^{-0,32}+N_{\text {obs }}\right),
\end{gathered}
$$

soit

$$
I_{N}=100 \times\left(4,8-4,8 \times M_{\mathrm{obs}}^{-0,32}+N_{\mathrm{obs}}\right) / 4,8 .
$$

\section{Phosphore}

Nous avons pris comme référence l'ajustement correspondant à l'ensemble des données de la parcelle $\mathrm{B}$, soit $P=0,24 N^{0,64}$ (d'après tableau III), où $N$ et $P$ sont les teneurs en pourcentage de la matière sèche. Nous avons calculé le rapport $P_{\text {obs }} / P_{\text {pot }}$, soit: $l p=P_{\text {obs }} / 0,24 N_{\text {obs }}{ }^{0,64}$ ou Ip $=100 \times 4,17 \mathrm{P}_{\text {obs }} \times \mathrm{N}_{\mathrm{obs}}{ }^{-0,64}$.

\section{Potassium}

Le même calcul a été réalisé avec la référence $K=1,62 N^{0,48}$ établie avec les données des parcelles $M$ et $\mathrm{T} \cdot I k=100 \times 0,62 K_{\text {obs }} \times$ Nobs ${ }^{-0,48}$.

Tout comme pour l'azote, il conviendra de s'assurer que des variations d'indices en cours de repousse ne proviennent pas de coefficients des courbes de référence sans valeur générale. Un indice égal à 100 indiquera un niveau de nutrition non limitant.

\section{CONCLUSION}

Avant de procéder à une validation des références proposées, on doit s'interroger sur les effets possibles de la nature des espèces ou de conditions de croissance particulières sur les teneurs en éléments minéraux.

La courbe de dilution de référence de $\mathrm{N}$ en fonction de la biomasse aérienne produite est 
quasiment la même pour de nombreuses espèces et variétés de graminées et légumineuses fourragères (Lemaire et al, 1989). Nos résultats recueillis dans plusieurs prairies permanentes différant par leur composition botanique le confirment, à condition que les prélèvements d'herbe soient réalisés avant sénescence importante. De nombreuses études ont eu lieu pour établir un lien entre la teneur des différentes espèces de végétaux en $P$ et $K$ et la richesse du substrat en ces mêmes éléments. Dans ce cas, même pour un stade phénologique identique, il apparaît de fortes différences de teneurs entre espèces d'une parcelle donnée (Lambert et al, 1973). Mais pour les interpréter, il nous semble important de rapporter les écarts de teneurs aux différences de biomasse aérienne et donc de dilution. Des comparaisons de teneurs en $P$ et $K$ de trèfle blanc séparé des autres espèces de plusieurs prairies permanentes montrent la vraisemblance de cette dernière hypothèse (Duru, non publié).

Si l'on veut porter un diagnostic fiable, on doit établir dans quelle mesure il est indépendant des conditions de croissance. II est connu que le régime hydrique modifie la dynamique d'absorption des minéraux, que ce soit du fait d'un stress hydrique (Gardwood et Williams, 1967 pour $\mathrm{N}$; Cole et al, 1977 pour $P$ ) ou d'un excès d'eau (Glinski et Sepwienski, 1985). Étant donné les différences connues quant à l'absorption des différents éléments minéraux, les relations entre leurs teneurs seront changées, et il convient donc de s'affranchir de ces conditions de croissance particulières.

Ces réserves étant faites, les indices de nutrition proposés doivent être validés à partir de données recueillies sur d'autres sites que celui ayant servi à les élaborer. Dans une deuxième partie, on s'attachera tout particulièrement à interpréter leur variation au cours d'une repousse et à comparer les informations qu'ils fournissent en regard des analyses chimiques de terre.

\section{REMERCIEMENTS}

Nous tenons à remercier $\mathrm{M}$ Bosc, B Colomb, JC Fardeau, A Langlet, $\mathrm{G}$ Lemaire et FX de Montard pour leur aide lors de la rédaction de ce manuscrit.

\section{RÉFÉRENCES}

Balent G, Duru M (1984) Influence des modes d'exploitation sur les caractéristiques et l'évolution des surfaces pastorales. Agronomie 4, 113124

Bates TE (1971) Factors affecting critical nutrient concentrations in plants and their evaluation: a review. Soil Sci 112, 116-130

Beaufils ER (1973) Diagnosis and recomendation integrated system (DRIS). Soil Sci Bull (1), Univ Natal, S Africa, 1-132

Bosc M, Maertens C (1981) Rôle de l'accroissement du système racinaire dans l'absorption de divers états du potassium du sol. Agrochimica $25,1-8$

Callot G, Chamayou H, Maertens C, Salsac L (1982) Incidence sur la nutrition minérale. In: Les interactions sol-racine INRA, $325 p$

Calvet R (1968) Analyse du concept de biodisponibilité d'une substance dans le sol. Sci Sol 26, 183-202

Claasen N, Syring KM, Jungk A (1986) Verification of a mathematical model by simulating potassium uptake from soil. Plant Soil 95, 209-220

Cole CV, Innis GS, Stewart JWB (1977) Simulation of phosphorus cycling in semiarid grasslands. Ecology 58, 1-15

Duru M, Gibon A (1989) Étude de quelques facteurs de variation de la digestibilité de prairies permanentes durant la pousse printanière. XVI Int Grass Cong Nice, France, 865-866

Garwood EA, Williams TE (1967) Growth, water use and nutrient uptake from the subsoil by grass sward. J Agric Sci Camb 69, 125-130

Gillet M (1980) Les graminées fourragères, Coll Nature et Agriculture, Gauthier-Villars, $306 \mathrm{p}$

Glinski J, Stepniewski N (1985) Soil aeration and its role for plants, CRC Press, $229 p$

Lambert J, Denudt G, Van Oudenhove C (1973) Aspects écologiques et phytosociologiques de l'analyse minérale des herbages. Rev Agric 4, 893-908

Lemaire G, Salette J (1984) Relation entre dynamique de croissance et dynamique de prélèvement d'azote pour un peuplement de graminées fourragères. I. Étude de l'effet du milieu. Agronomie 4, 423-440

Lemaire G, Gruz P, Gosse G, Chartier M (1985) Étude des relations entre la dynamique de prélèvement d'azote et la dynamique de croissance en matière sèche d'un peuplement de luzerne. Agronomie 5, 685-692

Lemaire G, Gastal F, Salette J (1989) Analysis of the effect of $N$ nutrition on dry matter yield of a sward by reference to potential yield and op- 
timum N content. XVI Int Grassland Cong Nice, France, 179-180

Lemaire G, Gastal F, Gruz P, Greenwood DJ, Draycott A (1990). Relationships between plant-N, plant mass and relative growth rate for $\mathrm{C}_{3}$ and $\mathrm{C}_{4}$ crops. In : First Congr Eur Soc Agron, Paris 5th-7th December, $2 p$

Martin WE, Matocha JE (1972) Plant analysis as an aid in the fertilization of forage crops. Soil Sci Soc Am J, 393-425

Montard FX (de) (1988) Pratique de la fumure de fond, phosphatée et potassique, sur prairie. Persp Agric, 127, 104-113

Pons Y, Lemaire G, Lafon E, Salette J (1989) Intensification des prairies des marais de l'Ouest. II. Fertilisation et méthodes de diagnostic de la nutrition minérale. Fourrages, 120, 367-382

Salette $J(1982)$ The role of fertilizers in improving herbage quality and optimization of its utilization. In: Proc 12th Congr Int Potash Instit Bern, 117-144

Salette J, Lemaire G (1981) Sur la variation de la teneur en azote des graminées fourragères pendant leur croissance: formulation d'une loi de dilution. CR Séances Acad Sc Paris, t 292, 875878

Salette J, Huche L (1989) The diagnosis of grassland mineral nutrient status through herbage analysis. In: XVI Int Grassland Cong, Nice, France, 65-66

Salette J, Huché L (1991) Diagnostic de l'état de nutrition minérale d'une prairie par l'analyse du végétal: principes, mise en œuvre, exemples. Fourrages 125, 3-18

Salette J, Dumas Y, Sobesky O (1973) Éléments d'écologie des herbages à Pangola dans divers milieux des Antilles françaises. II. Données sur les éléments minéraux chez Digitaria decumbens cultivé dans divers milieux. Agron Trop, 28, 833-845

Salette J, Lemaire G, Robichet J (1982) Nitrogen and mineral uptake during regrowth of pure grass sward. In: Proc 9th Gen Meet of EGF, (Reading ed) Br Grassland Soc, 165-170

Williams RD (1957) Growth and nutrition of timothy (Phleum pratense L). III. Absorption and distribution of nitrogen, phosphorus and potassium during the first year of regrowth. Ann Appl Biol $45,664-673$ 